OPEN ACCESS

Edited by:

Yu Shang,

North University of China, China

Reviewed by:

Lei Wang,

Peking Union Medical College Hospital (CAMS), China Ahmed Bahrani,

University of Kentucky, United States

*Correspondence:

Yong Xu

xuyong@sxmu.edu.cn

these authors have contributed equally to this work and share first authorship

Specialty section:

This article was submitted to Neuroimaging and Stimulation, a section of the journal Frontiers in Psychiatry

Received: 13 August 2021 Accepted: 27 September 2021 Published: 05 November 2021

Citation:

Wen $D$, Lang X, Zhang $H, L i Q$, Yin Q, Chen $Y$ and $X u Y$ (2021) Task and Non-task Brain Activation Differences for Assessment of Depression and Anxiety by fNIRS.

Front. Psychiatry 12:758092. doi: 10.3389/fpsyt.2021.758092

\section{Task and Non-task Brain Activation Differences for Assessment of Depression and Anxiety by fNIRS}

\author{
Dan Wen ${ }^{1,2 \dagger}$, Xuenan Lang ${ }^{1 \dagger}$, Hang Zhang ${ }^{2}$, Qiqi Li $^{1}$, Qin Yin ${ }^{1}$, Yulu Chen ${ }^{2}$ and Yong $X u^{1,2,3 *}$ \\ ${ }^{1}$ First Hospital of Shanxi Medical University, Taiyuan, China, ${ }^{2}$ Department of Psychiatry, First Hospital/First Clinical Medical \\ College of Shanxi Medical University, Taiyuan, China, ${ }^{3}$ Department of Mental Health, Shanxi Medical University, Taiyuan, \\ China
}

Diagnosis and treatment of the patients with major depression (MD) or the combined anxiety and depression (A\&D) depend on the questionnaire, sometimes accompanied by tasks such as verbal fluency task (VFT). Functional near infrared spectroscopy (fNIRS) is emerging as an auxiliary diagnostic tool to evaluate brain function, providing an objective criterion to judge psychoses. At present, the conclusions derived from VFT or rest (non-task) studies are controversial. The purpose of this study is to evaluate if task performs better than non-task in separating healthy people from psychiatric patients. In this study, healthy controls (HCs) as well as the patients with MD or A\&D were recruited ( $n=10$ for each group) to participate in the non-task and VFT tasks, respectively, and the brain oxygenation was longitudinally evaluated by using fNIRS. An approach of spectral analysis is used to analyze cerebral hemoglobin parameters (i.e., Oxy and Deoxy), characterizing the physiological fluctuations in the non-task and task states with magnitude spectrum and average power. Moreover, the standard deviation of oxygenation responses during the non-task was compared with the peak amplitude during the task, with the aim to explore the sensitivity of the VFT task to brain activation. The results show that there is no significant difference $(p>0.05)$ among the three groups in average power during non-task. The VFT task greatly enhanced the magnitude spectrum, leading to significant difference $(p<0.05)$ in average power between any of two groups $(H C, M D$, and $A \& D)$. Moreover, 40\% patients with $A \& D$ have an intermediate peak (around $0.05 \mathrm{~Hz}$ ) in the magnitude spectrum when performing the VFT task, indicating its advantage in characterizing A\&D. We defined a rate of the non-task standard variation to the task peak amplitude (namely, SD-to-peak rate) and found that this rate is larger than $20 \%$ in $90 \%$ of the MD subjects. By contrast, only $40 \%$ HC subjects have an SD-to-peak rate larger than $20 \%$. These results indicate that the non-task may not be sufficient to separate MD or A\&D from HC. The VFT task could enhance the characteristics of the magnitude spectrum, but its intensity needs to be elevated so as to properly explore brain functions related to psychoses.

Keywords: functional near-infrared spectroscopy, non-task, verbal fluency task, anxiety, depression 


\section{BACKGROUND}

Depression and anxiety are two psychoses that affect millions of people in the world, and their comorbidity rate is high (1). Clinical symptoms and functional impairment are more severe in patients with the combined anxiety and depression $(A \& D)(2)$, and the major consequences include emotional, cognitive, and somatic symptoms. It has been found that the brain functions of $\mathrm{A} \& \mathrm{D}$ are different from either depression or anxiety alone. Patients with depression have the suppressed functions of brain regions that regulate emotion and cognition, thus exhibiting the relevant clinical symptoms $(3,4)$. By contrast, the alterations in cerebral cortex function occur in specific regions for $\mathrm{A} \& \mathrm{D}$ patients, particularly in the prefrontal lobe and temporal lobe, affecting execution, language, memory, and attention, as evidenced by the declined cognitive control ability (5).

Rapid advances in neurological imaging technologies, such as functional magnetic resonance imaging (fMRI) and electroencephalography (EEG), permit observation of the changes in both brain structure and function. Although fMRI could obtain functional parameters such as oxyhemoglobin index, the high cost precludes its routine use in psychiatric studies. In addition, fMRI measurement is strictly limited by body posture, making it difficult to perform verbal or cognitive tasks simultaneously. EEG provides rapid assessment of neural activity in the cerebral cortex and has been widely utilized in cognition and autonomic control. However, EEG does not directly reflect brain metabolism.

Functional near-infrared spectroscopy (fNIRS) is a noninvasive, functional neuroimaging technique that could assess brain function by quantifying cerebral oxygenation at the microvasculature level (6-8). After more than 40 years of development, this technology has been used for evaluation of cerebral function (9-11) and extended to the clinical field of psychiatry, as one of the objective modalities for probing psychoses (12). fNIRS is sensitive to external stimulation or events; it allows for longitudinal monitoring of the changes in brain metabolism. Hence, fNIRS is frequently adopted for brain functional assessment when various tasks are performed (13).

The non-task (i.e., doing nothing) is a resting state that is easy to implement. The spontaneous brain activity during non-task period can be used as a baseline reference for brain activation (14). In EEG studies, the features in the resting state were found to be associated with a variety of neuropsychiatric disorders (15). fMRI can explore brain function by measuring the temporal variations of blood oxygenation level dependence (BOLD) as well as the fluctuations in resting spontaneous neural activity (1618). Besides these, the spectral analysis of fNIRS permits the assessment of the autoregulation of brain function. The nontask does not generate motion artifacts and the obtained fNIRS signal is more reliable, with minimal signal noise. Recently, minor brain damage in the early stage of Alzheimer's was detected from the non-task EEG-fNIRS data (19). On the other hand, however, non-task does not challenge the brain function, which can only be realized by task paradigm (20).
The verbal fluency task (VFT) is a simple task that is widely used to assess language extraction and processing abilities (21, 22). fNIRS was also applied on the VFT task to distinguish different types of psychoses (23). In some studies, major depression (MD) patients were found to have the reduced responses in the orbitofrontal cortex (OFC) and frontal cortex (24), and the activation level is associated with the severity of MD symptoms (25). The schizophrenia patients were also found to have the reduced responses in the dorsolateral prefrontal cortex (DLPFC) (26), and bipolar disorder patients have the same performance in the left inferior frontal gyrus (IFG) (27). Under the VFT task, the prefrontal lobe is well activated (28), when compared with the non-task. In some fNIRS studies, it was found that the damaged cerebral cortex can be detected from both the non-task and the task. Hence, fNIRS technology is sensitive to both cognitive tasks and static state. However, task and non-task have not been extensively compared thus far.

Therefore, the comparison of cortex oxygen responses between VFT task and non-task will contribute to the detection of psychoses such as MD and A\&D. In addition, an in-depth analysis of the response pattern of brain oxygenation variables in healthy and psychiatric populations would effectively evaluate and further optimize fNIRS for the detection of psychoses (29).

\section{MATERIALS AND METHODS}

\section{Participants}

In this study, 10 patients with $\mathrm{MD}$ and 10 patients with $\mathrm{A} \& \mathrm{D}$ diagnosed according to the Diagnostic and Statistical Manual of Mental Disorders-V (DSM-V) (30) criterion were recruited at the outpatient clinic of the Department of Mental Health, First Hospital of Shanxi Medical University. The diagnosis was conducted by three experienced physicians according to the consistent criteria. Ten healthy controls (HCs) were recruited from the local community. The subjects were in the age range between 15 and 55 years. The average age of the subjects for MD, $\mathrm{A} \& \mathrm{D}$, and $\mathrm{HC}$, represented by mean \pm standard derivation, are $29.8 \pm 11.8,30.0 \pm 11.4$, and $31.8 \pm 11.1$, respectively. Every two of the three groups are aged-matched $(p>0.05)$. The time-course changes in cerebral cortex oxygenation during the VFT task and non-task were measured and analyzed among the three groups of subjects. All subjects have education level of high school level or above, and those who have neuropathy, severe physical diseases, substance abuse, or high suicide risk were excluded. The study was approved by the Ethics Committee of the First Hospital of Shanxi Medical University. After the study design was explained, all of the participants signed the consent form.

\section{Methods}

\section{fNIRS Data Acquisition}

The oxygenation data were collected by the Hitachi ETG4100 fNIRS instrument $(52 \mathrm{CH})$ at the First Hospital of Shanxi Medical University. The subjects completed the non-task and VFT protocol individually. Prior to experiments, the subject wore a helmet embedded with numerous near-infrared light sensors, covering the prefrontal region and both temporal lobes. The near-infrared light sensors were placed over the scalp according 
to the 10-20 international system. Each channel of the nearinfrared signals is collected from a source-detector (S-D) pair, at the separation of $3.0 \mathrm{~cm}$. A total of $11 \mathrm{~S}-\mathrm{D}$ channels are located on the prefrontal lobe, and $20 \mathrm{~S}-\mathrm{D}$ channels are located on the right and left temporal lobes. For each S-D channel, the near-infrared light is injected from the two laser sources (695 and $830 \mathrm{~nm}$ ) alternately into the human scalp. The reflected signals from the brain cortex are collected by the optical detector and used to calculate the oxygenation responses. The laser power $(8 \mathrm{~mW})$ is sufficiently low so as to be approved for clinical usage. Both prefrontal lobe and temporal lobes are reported to be activated to the VFT task. For all the subjects, the oxygenation data obtained from each channel were averaged over the prefrontal lobe and temporal lobes, yielding the average responses to the VFT task and non-task respectively.

Firstly, the 160-s non-task data were collected from all subjects. The non-task requires the participant to sit in a chair, open their eyes and look forward, and repeatedly count 1 through 10, with the thumbs and index fingers of both hands performing counter-finger movements in rhythm until the end of the task (31). The subject was then asked to rest for $5 \mathrm{~min}$. Subsequently, 160-s VFT task data were collected from all subjects. The fNIRS signals were collected for $30 \mathrm{~s}$ as the baseline prior to the task. During the VFT task, the subject was asked to construct the phrase with the simple words such as sky, earth, and human, lasting for $60 \mathrm{~s}(32,33)$. The post-task fNIRS signals were collected for $70 \mathrm{~s}$, repeatedly counting from 1 through 10. The cerebral oxygenation variables at each channel, including concentration of oxygenated hemoglobin (Oxy) and deoxygenated hemoglobin (Deoxy), were calculated from fNIRS signals according to the Beer-Lambert law. These variables at different channels were then averaged over the prefrontal region and both temporal lobes, yielding the cortex responses to the task or non-task.

\section{Data Analysis}

The spectral analysis was used to investigate the time-course oxygenation variables (Oxy and Deoxy) curves, yielding the relationship between frequency and the magnitude of spectrum (34-36). This spectrum relationship is to characterize the oxygenation oscillations of the three populations (MD, A\&D, and $\mathrm{HC}$ ). Specifically, the Oxy/Deoxy data were collected using the fNIRS instrument at the sample time of $0.1 \mathrm{~s}$, leading to a total of 1,600 data points over the 160-s measurement period. These time-course curves were preprocessed by a Hanning window to reduce the effect of spectral leakage. Then, fast Fourier transform (FFT) was applied to transform the time-course curve into the frequency domain, from which the magnitude spectrum of the same length was obtained over the $0.01-16.0-\mathrm{Hz}$ range, at the sample rate of $0.01 \mathrm{~Hz}$. The average power, defined as the average of magnitude square over the whole spectrum $(0.01-16.0 \mathrm{~Hz})$, was calculated for each individual. In addition, the rate between the standard deviation of the Oxy/Deoxy curve during non-task and the peak amplitude during VFT task was calculated, in order to explore the sensitivity of the VFT task to brain activation. Furthermore, the Bland-Altman analysis is adopted to assess the dispersion of the intra-group data, and the threshold is set as 1.96 times of standard deviation (SD).

\section{RESULTS}

\section{Comparison of the Rate of Brain Activation Between Non-task and VFT Task}

Figure 1 shows the rate of the Oxy/Deoxy standard deviation during the non-task to the maximal change (i.e., peak amplitude) during the VFT task, namely, SD-to-peak rate, for the three groups. It can be seen that $90 \%$ of the MD patients had an SD-to-peak rate larger than 20\% (Figure 1A), while $40 \%$ of the HCs have a rate larger than 20\% (Figure 1B). As for the $A \& D$ patients, $60 \%$ of the group have a rate larger than $20 \%$ (Figure 1C). Additionally, it is also found that the intra-subject variability of this rate was large in each group of subjects, and no significant differences were found between groups. Regression analyses indicate that the age is not relevant $(p>0.05)$ to the NIRS outcomes (i.e., Oxy and Deoxy).

\section{Distribution in the Degree of Brain Activation in the Non-task State}

Figure 2 exhibits the Bland-Altman analysis of the Oxy integral values by the non-task. As seen clearly, $10 \%(1 / 10)$ of the data points are outside the limit of 1.96 times the standard deviation, regardless of the group (i.e., $\mathrm{MD}, \mathrm{HC}$, or $\mathrm{A} \& \mathrm{D}$ ). The BlandAltman analysis of the Deoxy exhibits a similar conclusion. These results indicate that the healthy and mental-disordered populations have similar intra-subject variability.

\section{The Difference Between Non-task and VFT Task in the Oxy Magnitude Spectrum}

The average magnitude spectra of Oxy time-course curves during non-task and VFT task in the three groups are shown in Figures 3, 4, respectively. Under non-task, it can be seen that the magnitude decreased with the increase in frequency (Figure 3). The groups in magnitude order from largest to the smallest were $\mathrm{HC}, \mathrm{A} \& \mathrm{D}$, and $\mathrm{MD}$. The average magnitude during the VFT task were substantially higher than that during the nontask (around twice), while the order remained the same (i.e., HC, $\mathrm{A} \& \mathrm{D}$, and $\mathrm{MD})$.

The Oxy average power (represented by mean \pm standard derivation) during non-task is $4.8 \pm 9.3,1.7 \pm 1.3$, and $3.2 \pm 5.1$ $\left(10^{-2} \cdot \mathrm{mm} \cdot \mathrm{Mm}\right)^{-2}$ for $\mathrm{HC}, \mathrm{MD}$, and $\mathrm{A} \& \mathrm{D}$, respectively, and no significant difference $(p>0.05)$ was found among the groups. This average power was greatly enhanced during VTF task, reaching $14.0 \pm 9.8,2.9 \pm 3.4$, and $6.7 \pm 4.5$, respectively. Furthermore, a significant difference $(p<0.05)$ was found between any of the two groups (i.e., HC, MD, and A\&D).

\section{Characteristics of the Typical Magnitude Spectrum in the Task State}

We also found that the variability of the Oxy magnitude spectrum curve at the non-task state is small, regardless of the group. Hence, the average magnitude spectrum curve would wellrepresent the individual responses. By contrast, there is much 

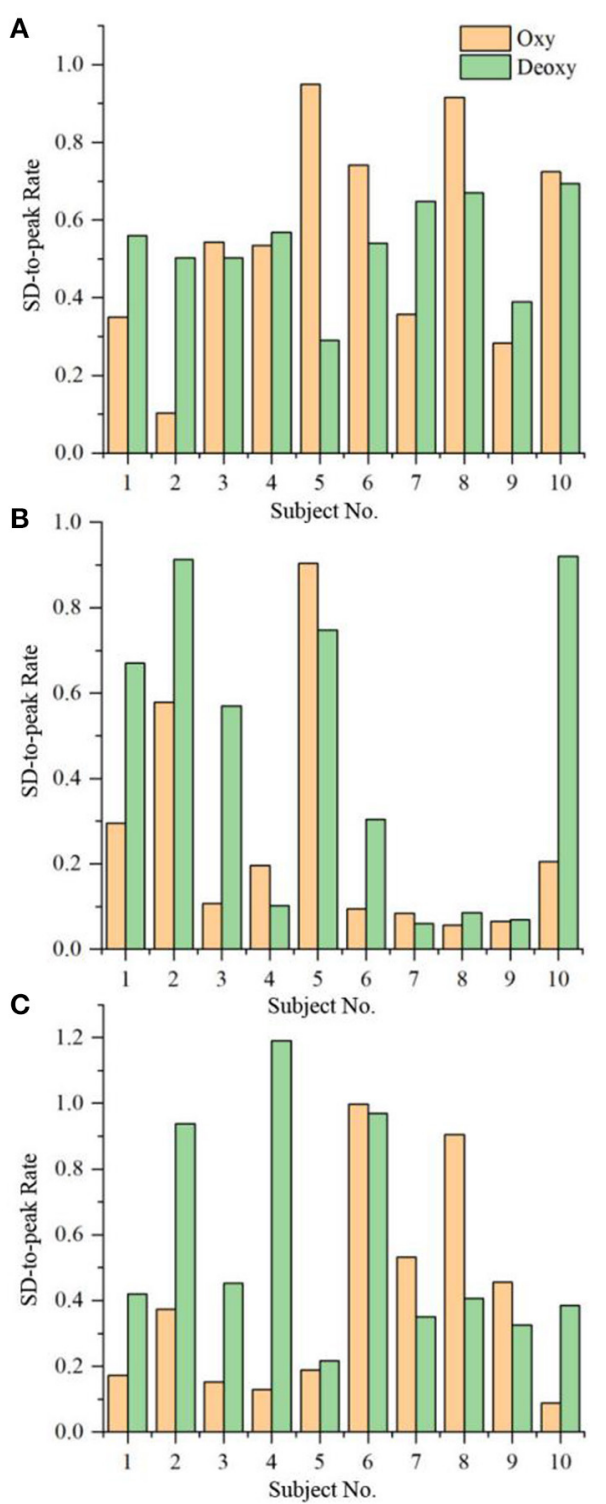

FIGURE 1 | The rate of the Oxy/Deoxy standard deviation during non-task to the maximal change (i.e., peak amplitude) during VFT task for the three groups, i.e. (A) MD, (B), $\mathrm{HC}$ and (C) A\&D

larger inter-subject variability in the Oxy magnitude spectrum when the VFT task was performed. Figure 5 shows the typical Oxy magnitude spectrum curve from a representative individual in each group. The MD exhibits the magnitude spectrum curve similar to that of $\mathrm{HC}$, with a small peak value around $0.05 \mathrm{~Hz}$ (Figures 5A,B). By contrast, four of the $10 \mathrm{~A} \& \mathrm{D}$ showed a strong intermediate peak around $0.05 \mathrm{~Hz}$ (Figure 5C). This is because not all of the A\&D patients had such strong intermediate peak, whose frequency also varied in a range (i.e., not the same frequency). Therefore, the average spectrum curve of the A\&D population did not clearly exhibit the strong intermediate peak. Nevertheless, none of the $\mathrm{HC}$ and MD groups were found to have such a special feature.

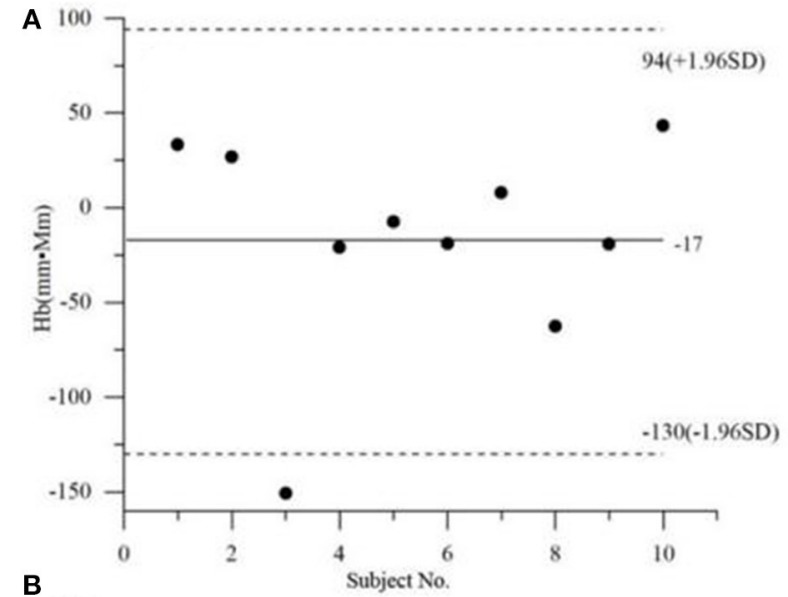

B
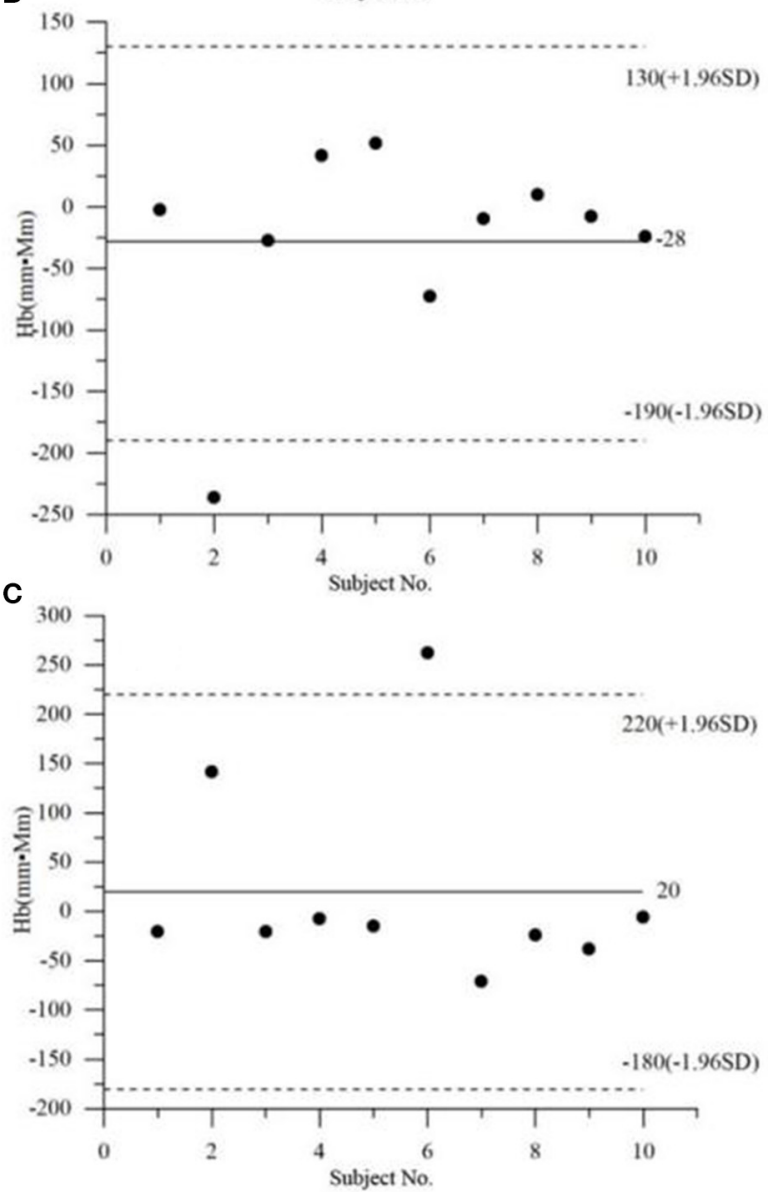

FIGURE 2 | Bland-Altman analysis of the Oxy integral values (i.e., area of the Oxy curve) by the non-task, in the three groups, i.e., (A) MD, (B) HC, and (C) A\&D.

\section{DISCUSSION}

fNIRS is an easy-to-use and low-cost technology for assessing brain function that is associated with cerebral metabolism. This technology has also been used as the auxiliary tool for 

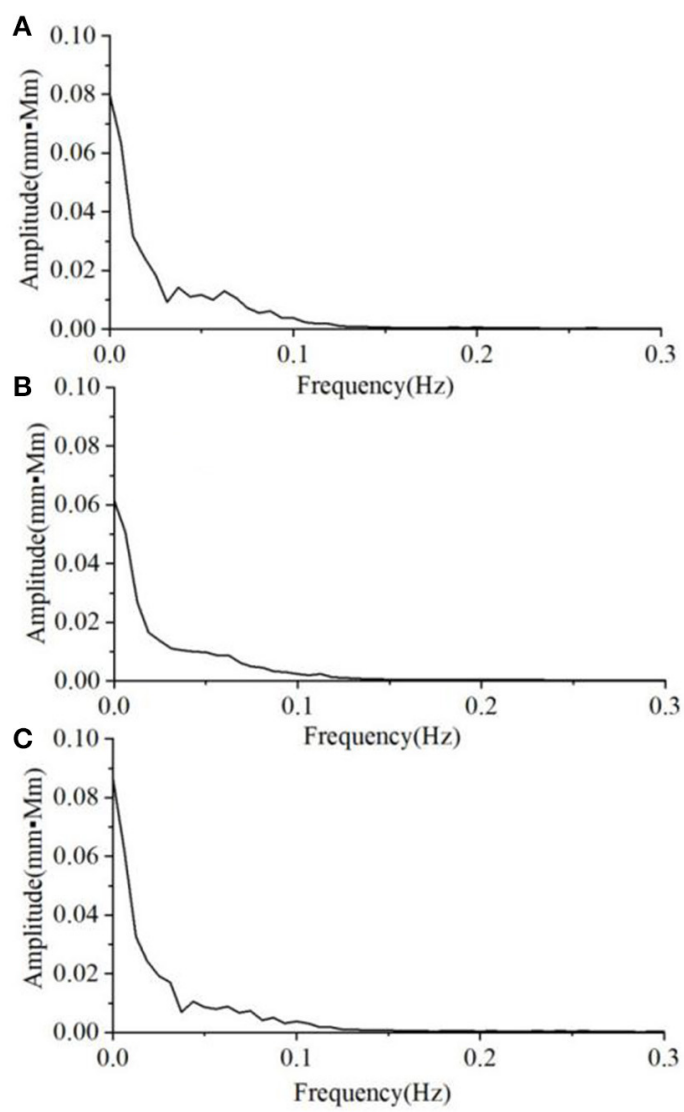

FIGURE 3 | The average magnitude spectrum of the Oxy time-course curves during non-task, in the three groups, i.e. (A) HC, (B) MD, and (C) A\&D. Note that the magnitude is almost zero at the range of $0.3-16.0 \mathrm{~Hz}$ and not shown in the figure.

diagnosis of psychoses (37). For example, the fNIRS was used to assess the autoregulation capacity of the brain cortex, which may be affected by psychoses. The protocols of non-task or task were adopted for this assessment $(38,39)$. The specificity and sensitivity of psychiatric diagnosis relies on several factors including the detection techniques, experiment protocols, and stimulus intensity. At present, the conclusions derived from VFT or rest (non-task) studies are controversial, and no oxygenation parameters were claimed to diagnose the psychiatric diseases. Several factors might account for this reason, such as the task intensity and duration for evoking the brain cortex, the intrasubject variability, and the oscillation characteristics related to the mental disorders. This study is designed to explore if the VFT task is strong enough to challenge the brain cortex, when compared with the rest state. We also investigated if the oscillation characteristics, which are generally evaluated by the rest state, could be affected by the VFT task.

A total of 30 subjects were recruited to participate in this study, with 10 for each group (HC, MD, and A\&D). The sample size $(n=10)$ might not be large enough to observe the statistical difference. Nevertheless, the similar sample size $(n=6-12)$ is
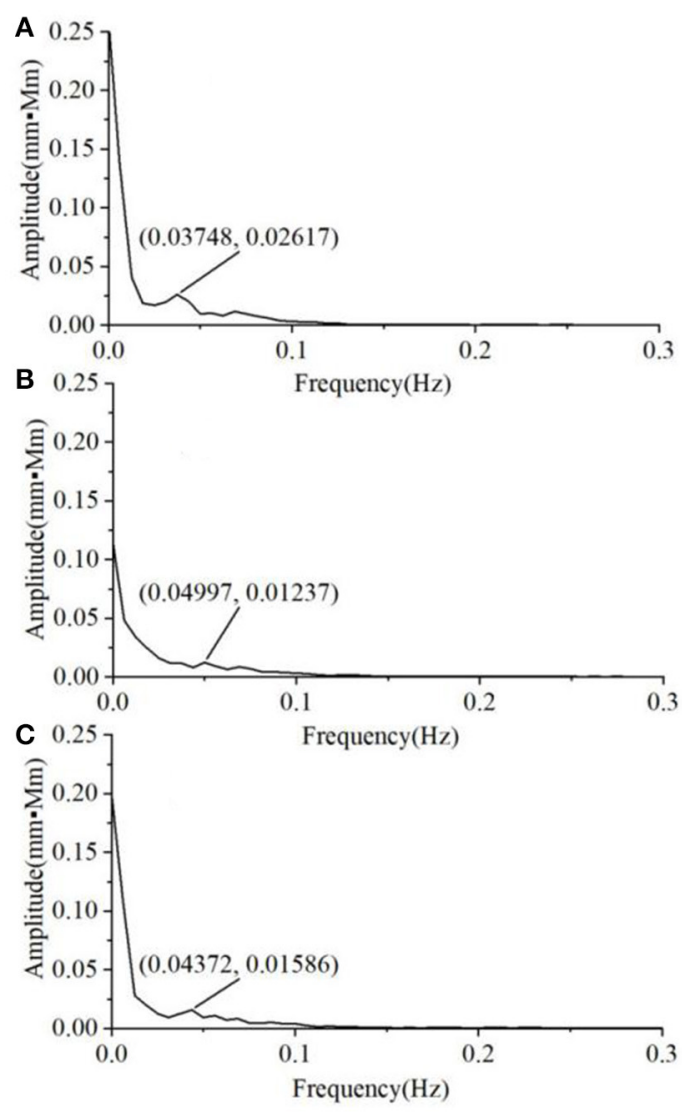

FIGURE 4 | The average magnitude spectrum of the Oxy time-course curves during the VFT task in the three groups, i.e. (A) HC, (B) MD, and (C) A\&D. Note that the magnitude is almost zero at the range of $0.3-16.0 \mathrm{~Hz}$ and not shown in the figure.

often reported in the NIRS studies exploring the brain cortex functions, along with statistical analysis results (40-44). The sample size is limited to the subject's availability and willingness to participate in the study. More subjects will be recruited and the advanced analysis algorithms will be explored, which is one objective of our future works.

A comparative study on brain oxygenation response by task and non-task was conducted on the 30 subjects. VFT was chosen as the task model because it is most widely used in psychiatric evaluation, and the changes in the brain oxygenation were found during the task period. Moreover, we assessed the task and non-task states in both amplitude change and spectrum. Previous studies have shown that MD patients have the reduced brain metabolism and task responses, indicating a cognitive impairment (45). Furthermore, the insufficient performance of MD patients was also observed when conducting the VFT task (46).

In order to evaluate the brain oxygenation activation by the VFT task, an index of SD-to-peak was calculated for each subject. We found that $90 \%$ of MD patients have a rate larger than $20 \%$, indicating that $\mathrm{MD}$ is associated with the deficiency in brain 

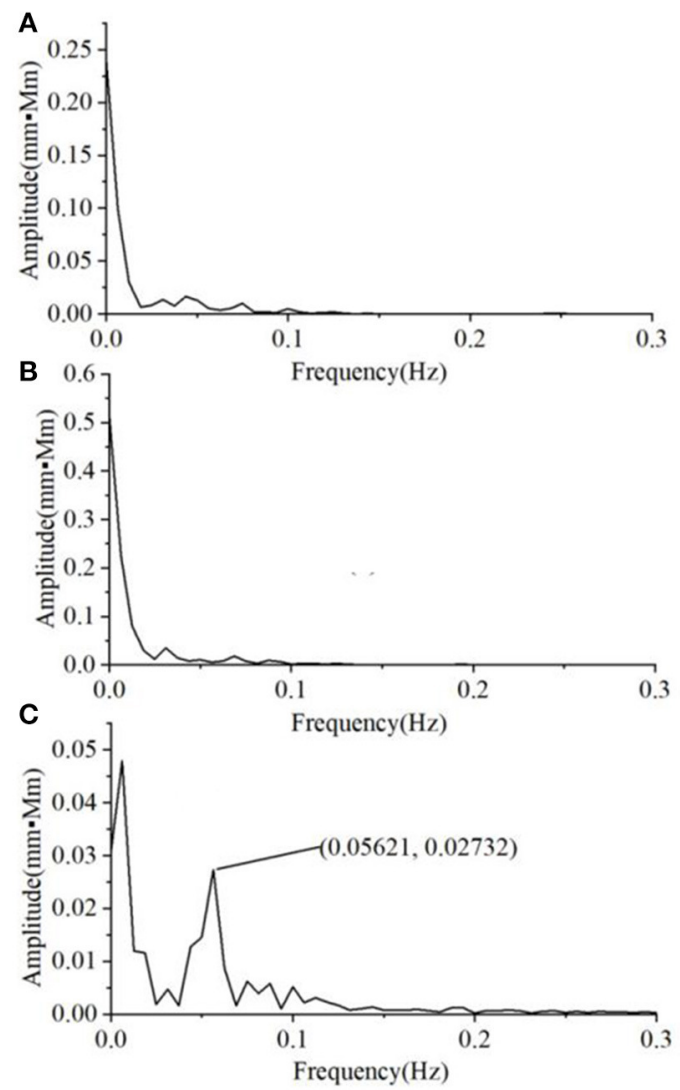

FIGURE 5 | The typical Oxy magnitude spectrum curve from a representative individual in each of three groups, i.e. (A) HCS, (B) MD, and (C) A\&D. Note that the magnitude is almost zero at the range of $0.3-16.0 \mathrm{~Hz}$ and not shown in the figure.

oxygen activation by the VFT task, which would be a marker of depressive state. By contrast, this rate is larger than $20 \%$ in $40 \% \mathrm{HC}$ and $60 \% \mathrm{~A} \& \mathrm{D}$ populations, respectively (Figure 1), indicating that majority of $A \& D$ responded positively to the VFT task. These observations exhibit that fNIRS has the potential to separate the depression through the brain oxygenation activation, if proper task is implemented.

With the approach of Bland-Altman to analyze the distribution of the Oxy integral values in $\mathrm{MD}, \mathrm{HC}$, and $\mathrm{A} \& \mathrm{D}$, we found that $10 \%(1 / 10)$ of the data points are outside of the 1.96 times the standard deviation (Figure 2), regardless of the group. This observation demonstrates that MD, HC, and $\mathrm{A} \& \mathrm{D}$ are similar in terms of individual data distribution.

As for the spectral analysis, we found that the magnitude is decreased with the increase in frequency. For each individual, the magnitude spectrum was mainly focused at $0.05-0.1 \mathrm{~Hz}$, indicating low-frequency oscillations in the spontaneous state. Under the non-task protocol, no significant difference was found among the three groups (Figure 3), indicating that static status would not alter the characteristic of low-frequency oscillation.

When performing the VFT task, we found that the magnitude of spectrum was substantially enhanced for all the individuals, with $\mathrm{HC}$ being the highest, $\mathrm{A} \& \mathrm{D}$ the second, and $\mathrm{MD}$ the lowest. In the past, it is difficult to separate $\mathrm{MD}$ and $\mathrm{A} \& \mathrm{D}$ by using fNIRS or other neuroimaging modalities. In this study, 40\% A\&D patients were found to show a strong intermediate peak (around $0.05 \mathrm{~Hz}$ ) in the magnitude spectrum when performing the VFT task, while this strong intermediate peak was not found in HC and MD populations. Although the underlying mechanism for this difference is unclear, this observation is beneficial for future diagnosis of the $\mathrm{A} \& \mathrm{D}$ disease.

Our spectral analyses of oxygenation data verified this hypothesis, i.e., the magnitude during the VFT task is much higher than that during the non-task status. We also observed that the Oxy average power has no significant difference among the three groups during non-task. By contrast, a significant difference was found between any of the two groups. These outcomes indicate that the parameter of average power, when enhanced by the VFT task, also characterizes well the diseased populations.

The aim of the repeated number counting and rhythm finger movement is to enforce the subjects to concentrate on this tedious task and minimize the intra-subject variability resulting from random thinking. According to the literature (47), the finger movement would primarily evoke the activities in the parietal lobe, rather than the prefrontal lobe and temporal lobes that were measured with fNIRS in this study. Hence, the fNIRS signals originating from finger movement can be considered as a small portion of the baseline data.

Nevertheless, the baseline data (including those from number counting and finger movements) may also affect the data analyses. The potential solution for minimizing the baseline effect would be increasing the task intensity (e.g., VFT duration) as well as utilizing the advanced algorithms (e.g., independent component analysis-ICA) to separate the baseline signals, which will be one objective of our future works.

As the summary, the outcomes derived from this study demonstrate that the standard VFT $(60 \mathrm{~s})$ might not be able to evoke sufficient oxygenation changes in the brain cortex, as evidenced by the $>20 \%$ SD-to-peak rate in the majority of subjects, especially in MD patients. We also found that the VFT might enhance the oscillation characteristics of Oxy curves. The intra-subject variability is similar among the three groups. All of these discoveries have not been reported in the previous studies. We believe that the fusion of multiple parameters, rather than the single parameter, would better localize the target diseases (MD or A\&D), along with the advanced algorithm (e.g., artificial intelligent algorithm). The conclusion derived from this study might be helpful in future exploring the advanced diagnostic approaches.

\section{CONCLUSIONS}

To conclude, fNIRS is an easy-to-use technology for longitudinally monitoring brain function (48), and its efficiency for psychosis diagnosis relies on the physiological protocol. In this study, we used fNIRS to compare between task and nontask states in brain oxygenation activation and to explore the 
characteristic markers of MD and A\&D. We found that the MD is associated with the deficient activation in brain oxygenation, evidenced by the relatively large SD-to-peak rate. In addition, $\mathrm{A} \& \mathrm{D}$ is characterized by the intermediate peak in spontaneous low-frequency oscillations. Nevertheless, the precise diagnosis of $\mathrm{MD}$ and $\mathrm{A} \& \mathrm{D}$ is negatively affected by the intra-subject variability. The optimal tasks, with small intra-subject variability and the enhanced oxygenation activation, need to be developed to further explore the brain functions associated with psychoses $(13,49)$.

\section{DATA AVAILABILITY STATEMENT}

The original contributions presented in the study are included in the article/supplementary material, further inquiries can be directed to the corresponding authors.

\section{ETHICS STATEMENT}

The studies involving human participants were reviewed and approved by the Ethics Committee of the First Hospital of Shanxi Medical University. Written informed consent to participate in this study was provided by the participants' legal guardian/next of kin.

\section{REFERENCES}

1. Kessler RC, Sampson NA, Berglund P, Gruber MJ, Al-Hamzawi A, Andrade $\mathrm{L}$, et al. Anxious and non-anxious major depressive disorder in the World Health Organization World Mental Health Surveys. Epidemiol Psychiatr Sci. (2015) 24:210-26. doi: 10.1017/S2045796015000189

2. Crane NA, Jenkins LM, Dion C, Meyers KK, Weldon AL, Gabriel LB, et al. Comorbid anxiety increases cognitive control activation in major depressive disorder. Depress Anxiety. (2016) 33:967-77. doi: 10.1002/da.22541

3. Atique-Ur-Rehman H, Neill JC. Cognitive dysfunction in major depression: From assessment to novel therapies. Pharmacol Ther. (2019) 202:5371. doi: 10.1016/j.pharmthera.2019.05.013

4. Kondo A, Shoji Y, Morita K, Sato M, Ishii Y, Yanagimoto H, et al. Characteristics of oxygenated hemoglobin concentration change during pleasant and unpleasant image-recall tasks in patients with depression: comparison with healthy subjects. Psychiatry Clin Neurosci. (2018) 72:61122. doi: $10.1111 /$ pcn. 12684

5. MacNamara A, Proudfit GH. Cognitive load and emotional processing in generalized anxiety disorder: electrocortical evidence for increased distractibility. J Abnorm Psychol. (2014) 123:557-65. doi: 10.1037/a0036997

6. Akashi H, Tsujii N, Mikawa W, Adachi T, Kirime E, Shirakawa O. Prefrontal cortex activation is associated with a discrepancy between selfand observer-rated depression severities of major depressive disorder: a multichannel near-infrared spectroscopy study. J Affect Disord. (2015) 174:165-72. doi: 10.1016/j.jad.2014.11.020

7. Bai J, Zhu Q, Liu Y, Zhou Y, Shi T, Gui Z, et al. PV-MBLL algorithm for extraction of absolute tissue oxygenation information by diffuse optical spectroscopy. Comput Methods Programs Biomed. (2020) 193:105456. doi: 10.1016/j.cmpb.2020.105456

8. Li T, Lin Y, Shang Y, He L, Huang C, Szabunio M, et al. Simultaneous measurement of deep tissue blood flow and oxygenation using noncontact diffuse correlation spectroscopy flow-oximeter. Sci Rep. (2013) 3:1358. doi: 10.1038/srep01358

9. Bahrani AA, Kong W, Shang Y, Huang C, Smith CD, Powell DK, et al. Diffuse optical assessment of cerebral-autoregulation in older

\section{AUTHOR CONTRIBUTIONS}

DW and XL conceived of and led on the study design, managed the literature searches, and undertook the statistical analysis, under the supervision of $\mathrm{YX}$, and wrote the first draft. $\mathrm{HZ}$ and YC made the subsequent revisions of the manuscript. QL and QY contributed to collecting data. All authors contributed to and have approved the final manuscript.

\section{FUNDING}

This study was financially supported by the National Natural Science Foundation of China (81971601 and 81701326), the National Key Research and Development Program of China (2016YFC1307004), and the Multidisciplinary Team for Cognitive Impairment of the Shanxi Science and Technology Innovation Training Team (201705D131027).

\section{ACKNOWLEDGMENTS}

We thank Shanxi Hengtai-yifeng Science and Technology Co., Ltd., for the data processing and Dr. Zhongshi Jin for critically proofreading the manuscript. adults stratified by cerebrovascular risk. J Biophotonics. (2020) 13:e202000073. doi: 10.1002/jbio.202000073

10. Pan B, Huang $C$, Fang $X$, Huang $X, L i ~ T$. Noninvasive and sensitive optical assessment of brain death. J Biophotonics. (2019) 12:e201800240. doi: 10.1002/jbio.201800240

11. Zhao M, Mazdeyasna S, Huang C, Agochukwu-Nwubah N, Bonaroti A, Wong L, et al. Noncontact speckle contrast diffuse correlation tomography of blood flow distributions in burn wounds: a preliminary study. Mil Med. (2020) 185:82-7. doi: 10.1093/milmed/usz233

12. Plewnia C, Schroeder PA, Wolkenstein L. Targeting the biased brain: noninvasive brain stimulation to ameliorate cognitive control. Lancet Psychiatry. (2015) 2:351-6. doi: 10.1016/S2215-0366(15)00056-5

13. Nishizawa Y, Kanazawa T, Kawabata Y, Matsubara T, Maruyama S, Kawano $\mathrm{M}$, et al. fNIRS assessment during an emotional stroop task among patients with depression: replication and extension. Psychiatry Investig. (2019) 16:806. doi: 10.30773/pi.2018.11.12.2

14. Smitha KA, Akhil Raja K, Arun KM, Rajesh PG, Thomas B, Kapilamoorthy TR, et al. Resting state fMRI: a review on methods in resting state connectivity analysis and resting state networks. Neuroradiol J. (2017) 30:30517. doi: $10.1177 / 1971400917697342$

15. Khanna A, Pascual-Leone A, Michel CM, Farzan F. Microstates in restingstate EEG: current status and future directions. Neurosci Biobehav Rev. (2015) 49:105-13. doi: 10.1016/j.neubiorev.2014.12.010

16. Elliott R, Lythe K, Lee R, McKie S, Juhasz G, Thomas EJ, et al. Reduced medial prefrontal responses to social interaction images in remitted depression. Arch Gen Psychiatry. (2012) 69:37-45. doi: 10.1001/archgenpsychiatry.2011.139

17. Strangman G, Culver JP, Thompson JH, Boas DA, A. quantitative comparison of simultaneous BOLD fMRI and NIRS recordings during functional brain activation. Neuroimage. (2002) 17:719-31. doi: 10.1006/nimg.2002.1227

18. Feige B, Spiegelhalder K, Kiemen A, Bosch OG, Tebartz van Elst L, Hennig J, et al. Distinctive time-lagged resting-state networks revealed by simultaneous EEG-fMRI. Neuroimage. (2017) $145(\mathrm{Pt}$ A):1-10. doi: 10.1016/j.neuroimage.2016.09.027

19. Chiarelli AM, Perpetuini D, Croce P, Filippini C, Cardone D, Rotunno L, et al. Evidence of Neurovascular Un-Coupling in Mild Alzheimer's Disease 
through Multimodal EEG-fNIRS and Multivariate Analysis of Resting-State Data. Biomedicines. (2021) 9:337. doi: 10.3390/biomedicines 9040337

20. Zou QH, Zhu CZ, Yang Y, Zuo XN, Long XY, Cao QJ, et al. An improved approach to detection of amplitude of low-frequency fluctuation (ALFF) for resting-state fMRI: fractional ALFF. J Neurosci Methods. (2008) 172:13741. doi: 10.1016/j.jneumeth.2008.04.012

21. Alvarez JA, Emory E. Executive function and the frontal lobes: a meta-analytic review. Neuropsychol Rev. (2006) 16:17-42. doi: 10.1007/s11065-006-9002-x

22. Fallgatter AJ, Roesler M, Sitzmann L, Heidrich A, Mueller TJ, Strik WK. Loss of functional hemispheric asymmetry in Alzheimer's dementia assessed with near-infrared spectroscopy. Brain Res Cogn Brain Res. (1997) 6:6772. doi: 10.1016/S0926-6410(97)00016-5

23. Wei Y, Chen Q, Curtin A, Tu L, Tang X, Tang Y, et al. Functional near-infrared spectroscopy (fNIRS) as a tool to assist the diagnosis of major psychiatric disorders in a Chinese population. Eur Arch Psychiatry Clin Neurosci. (2021) 271:745-57. doi: 10.1007/s00406-020-01125-y

24. Murray EA, Wise SP, Drevets WC. Localization of dysfunction in major depressive disorder: prefrontal cortex and amygdala. Biol Psychiatry. (2011) 69:e43-54. doi: 10.1016/j.biopsych.2010.09.041

25. Liu X, Sun G, Zhang X, Xu B, Shen C, Shi L, et al. Relationship between the prefrontal function and the severity of the emotional symptoms during a verbal fluency task in patients with major depressive disorder: a multichannel NIRS study. Prog Neuropsychopharmacol Biol Psychiatry. (2014) 54:114-21. doi: 10.1016/j.pnpbp.2014.05.005

26. Luo X, Qi Y, Wang H, Wang Y, He T, Rong B, et al. Prefrontal cortex dysfunction during verbal fluency task after atypicalantipsychotic treatment in schizophrenia: a near-infrared spectroscopy imaging study. Neurosci Lett. (2018) 686:101-5. doi: 10.1016/j.neulet.2018.09.001

27. Feng K, Law S, Ravindran N, Chen GF, Ma XY, Bo X, et al. Differentiating between bipolar and unipolar depression using prefrontal activation patterns: promising results from functional near infrared spectroscopy (fNIRS) findings. J Affect Disord. (2021) 281:476-84. doi: 10.1016/j.jad.2020.12.048

28. Herrmann MJ, Ehlis AC, Fallgatter AJ. Frontal activation during a verbalfluency task as measured by near-infrared spectroscopy. Brain Res Bull. (2003) 61:51-6. doi: 10.1016/S0361-9230(03)00066-2

29. Yamamuro K, Kimoto S, Iida J, Kishimoto N, Tanaka S, Toritsuka M, et al. Distinct patterns of blood oxygenation in the prefrontal cortex in clinical phenotypes of schizophrenia and bipolar disorder. J Affect Disord. (2018) 234:45-53. doi: 10.1016/j.jad.2018.02.065

30. Bhati MT. Defining psychosis: the evolution of DSM-5 schizophrenia spectrum disorders. Curr Psychiatry Rep. (2013) 15:409. doi: 10.1007/s11920-013-0409-9

31. Moreno-Sánchez M, Aznar-Casanova JA, Valle-Inclán F. Attention to Monocular Images Bias Binocular Rivalry. Front Syst Neurosci. (2019) 13:12. doi: $10.3389 /$ fnsys. 2019.00012

32. Tung H, Lin WH, Lan TH, Hsieh PF, Chiang MC, Lin YY, et al. Network reorganization during verbal fluency task in fronto-temporal epilepsy: a functional near-infrared spectroscopy study. J Psychiatr Res. (2021) 138:5419. doi: 10.1016/j.jpsychires.2021.05.012

33. Quan W, Wu T, Li Z, Wang Y, Dong W, Lv B. Reduced prefrontal activation during a verbal fluency task in Chinese-speaking patients with schizophrenia as measured by near-infrared spectroscopy. Prog Neuropsychopharmacol Biol Psychiatry. (2015) 58:51-8. doi: 10.1016/j.pnpbp.2014. 12.005

34. Cursi M, Meraviglia MV, Fanelli GF, Chiesa R, Tirelli A, Comi $\mathrm{G}$, et al. Electroencephalographic background desynchronization during cerebral blood flow reduction. Clin Neurophysiol. (2005) 116:2577-85. doi: 10.1016/j.clinph.2005.06.026

35. Malenfant S, Brassard P, Paquette M, Le Blanc O, Chouinard A, Nadeau V, et al. Compromised cerebrovascular regulation and cerebral oxygenation in pulmonary arterial hypertension. J Am Heart Assoc. (2017) 6:e006126. doi: 10.1161/JAHA.117.006126

36. Charlot K, Antoine-Jonville S, Moeckesch B, Jumet S, Romana M, Waltz $\mathrm{X}$, et al. Cerebral and muscle microvascular oxygenation in children with sickle cell disease: influence of hematology, hemorheology and vasomotion. Blood Cells Mol Dis. (2017) 65:23-8. doi: 10.1016/j.bcmd.2017. 03.015

37. Koike S, Satomura Y, Kawasaki S, Nishimura Y, Kinoshita A, Sakurada H, et al. Application of functional near infrared spectroscopy as supplementary examination for diagnosis of clinical stages of psychosis spectrum. Psychiatry Clin Neurosci. (2017) 71:794-806. doi: 10.1111/pcn.12551

38. Tomioka H, Yamagata B, Kawasaki S, Pu S, Iwanami A, Hirano J, et al. A longitudinal functional neuroimaging study in medicationnaïve depression after antidepressant treatment. PLoS ONE. (2015) 10:e0120828. doi: 10.1371/journal.pone.0120828

39. Fu L, Xiang D, Xiao J, Yao L, Wang Y, Xiao L, et al. Reduced prefrontal activation during the tower of london and verbal fluency task in patients with bipolar depression: a multi-channel NIRS study. Front Psychiatry. (2018) 9:214. doi: 10.3389/fpsyt.2018.00214

40. Metzger FG, Schopp B, Haeussinger FB, Dehnen K, Synofzik M, Fallgatter AJ, et al. Brain activation in frontotemporal and Alzheimer's dementia: a functional near-infrared spectroscopy study. Alzheimers Res Ther. (2016) 8:56. doi: 10.1186/s13195-016-0224-8

41. Yang J, Kanazawa S, Yamaguchi MK, Kuriki I. Cortical response to categorical color perception in infants investigated by near-infrared spectroscopy. Proc Natl Acad Sci USA. (2016) 113:2370-5. doi: 10.1073/pnas.1512044113

42. Plichta MM, Herrmann MJ, Baehne CG, Ehlis AC, Richter MM, Pauli P, et al. Event-related functional near-infrared spectroscopy (fNIRS): are the measurements reliable? Neuroimage. (2006) 31:116-24. doi: 10.1016/j.neuroimage.2005.12.008

43. Rizki EE, Uga M, Dan I, Dan H, Tsuzuki D, Yokota H, et al. Determination of epileptic focus side in mesial temporal lobe epilepsy using long-term noninvasive fNIRS/EEG monitoring for presurgical evaluation. Neurophotonics. (2015) 2:025003. doi: 10.1117/1.NPh.2.2.025003

44. Tomioka H, Yamagata B, Takahashi $\mathrm{T}$, Yano $\mathrm{M}$, Isomura AJ, Kobayashi $\mathrm{H}$, et al. Detection of hypofrontality in drivers with Alzheimer's disease by near-infrared spectroscopy. Neurosci Lett. (2009) 451:252-6. doi: 10.1016/j.neulet.2008.12.059

45. Ohtani T, Nishimura Y, Takahashi K, Ikeda-Sugita R, Okada N, Okazaki Y. Association between longitudinal changes in prefrontal hemodynamic responses and social adaptation in patients with bipolar disorder and major depressive disorder. J Affect Disord. (2015) 176:78-86. doi: 10.1016/j.jad.2015.01.042

46. Rajtar-Zembaty A, Sałakowski A, Rajtar-Zembaty J, Starowicz-Filip A. Executive dysfunction in late-life depression. Psychiatr Pol. (2017) 51:70518. doi: 10.12740/PP/OnlineFirst/63765

47. Rodríguez-Aranda C, Castro-Chavira SA, Espenes R, Barrios FA, Waterloo K, Vangberg TR. The role of moderating variables on BOLD fMRI response during semantic verbal fluency and finger tapping in active and educated healthy seniors. Front Hum Neurosci. (2020) 14:203. doi: $10.3389 /$ fnhum. 2020.00203

48. Chen WL, Wagner J, Heugel N, Sugar J, Lee YW, Conant L, et al. Functional near-infrared spectroscopy and its clinical application in the field of neuroscience: advances and future directions. Front Neurosci. (2020) 14:724. doi: 10.3389/fnins.2020.00724

49. Lang $\mathrm{X}$, Wen $\mathrm{D}, \mathrm{Li} \mathrm{Q}$, Yin $\mathrm{Q}$, Wang $\mathrm{M}, \mathrm{Xu}$ Y. fNIRS evaluation of frontal and temporal cortex activation by verbal fluency task and high-level cognition task for detecting anxiety and depression. Front Psychiatry. (2021) 12:690121. doi: 10.3389/fpsyt.2021.690121

Conflict of Interest: The authors declare that the research was conducted in the absence of any commercial or financial relationships that could be construed as a potential conflict of interest.

Publisher's Note: All claims expressed in this article are solely those of the authors and do not necessarily represent those of their affiliated organizations, or those of the publisher, the editors and the reviewers. Any product that may be evaluated in this article, or claim that may be made by its manufacturer, is not guaranteed or endorsed by the publisher.

Copyright $\odot 2021$ Wen, Lang, Zhang, Li, Yin, Chen and Xu. This is an open-access article distributed under the terms of the Creative Commons Attribution License (CC $B Y)$. The use, distribution or reproduction in other forums is permitted, provided the original author(s) and the copyright owner(s) are credited and that the original publication in this journal is cited, in accordance with accepted academic practice. No use, distribution or reproduction is permitted which does not comply with these terms. 